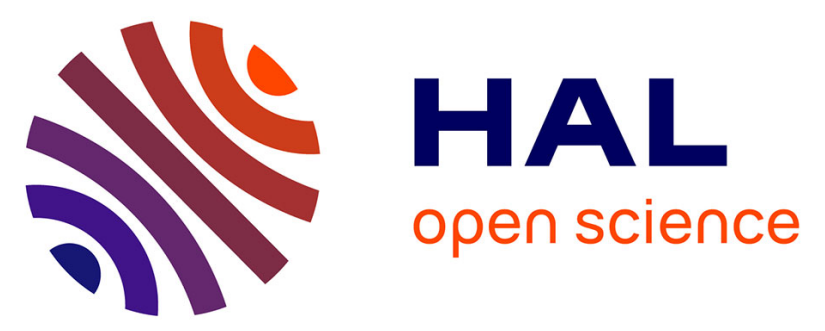

\title{
Chikungunya-Induced Arthritis in Reunion Island: A Long-Term Observational Follow-Up Study Showing Frequently Persistent Joint Symptoms, Some Cases of Persistent Chikungunya Immunoglobulin M Positivity, and No Anticyclic Citrullinated Peptide Seroconversion After 13 Years
}

Xavier Guillot, Anne Ribera, Philippe Gasque

\section{- To cite this version:}

Xavier Guillot, Anne Ribera, Philippe Gasque. Chikungunya-Induced Arthritis in Reunion Island: A Long-Term Observational Follow-Up Study Showing Frequently Persistent Joint Symptoms, Some Cases of Persistent Chikungunya Immunoglobulin M Positivity, and No Anticyclic Citrullinated Peptide Seroconversion After 13 Years. Journal of Infectious Diseases, 2020, 222, pp.1740 - 1744. 10.1093/infdis/jiaa261 . hal-03321874

\author{
HAL Id: hal-03321874 \\ https://hal.univ-reunion.fr/hal-03321874
}

Submitted on 18 Aug 2021

HAL is a multi-disciplinary open access archive for the deposit and dissemination of scientific research documents, whether they are published or not. The documents may come from teaching and research institutions in France or abroad, or from public or private research centers.
L'archive ouverte pluridisciplinaire HAL, est destinée au dépôt et à la diffusion de documents scientifiques de niveau recherche, publiés ou non, émanant des établissements d'enseignement et de recherche français ou étrangers, des laboratoires publics ou privés. 


\section{Chikungunya-Induced Arthritis in Reunion Island: A Long-Term Observational Follow-Up Study Showing Frequently Persistent Joint Symptoms, Some Cases of Persistent Chikungunya Immunoglobulin M Positivity, and No Anticyclic Citrullinated Peptide Seroconversion After 13 Years}

\author{
Xavier Guillot, ${ }^{1,2}{ }^{2}$ Anne Ribera, ${ }^{3,4}$ and Philippe Gasque ${ }^{2}$ \\ 1 Department of Rheumatology, Centre Hospitalier Universitaire de la Réunion, Saint-Denis, Reunion \\ Island, ${ }^{2} E P I$, Université de la Réunion, Saint-Denis, Reunion Island, ${ }^{3}$ Department of Internal Medicine, \\ Groupe Hospitalier Est de la Réunion, Saint-Benoit, Reunion Island, ${ }^{4}$ Department of Internal Medicine, \\ Centre Hospitalier Ouest de la Réunion, Saint-Paul, Reunion Island
}

Background. Reunion Island was struck by a massive Chikungunya outbreak in 2005-2006. Chikungunya infection is characterized by inflammatory joint symptoms, which may evolve into chronic arthritis.

Methods. In this long-term longitudinal observational monocentric study, after the 2005-2006 outbreak in Reunion Island, 159 patients were first referred to a rheumatologist for post-Chikungunya chronic musculoskeletal pain, 73 of them were diagnosed with classifiable Chikungunya-related chronic inflammatory rheumatic diseases ( $>3$ month symptom duration from the initial viral infection). Thirty of these 73 patients were clinically evaluated by a second rheumatologist in 2018-2019. The main objective of this second examination was to estimate the proportion of patients with persistent Chikungunya-related inflammatory joint symptoms after 13 years.

Results. Inflammatory joint symptoms persisted in $17 / 30$ patients after 13 years (therefore in at least $23.3 \%$ of the 73 patients initially diagnosed with Chikungunya-related inflammatory joint symptoms and $10.7 \%$ of the 159 patients referred for post-Chikungunya chronic musculoskeletal pain). In the symptom persistence subgroup, the prevalence of positive autoantibodies (antinuclear or ACPA) was significantly higher - without any seroconversion, Chikungunya IgG and IgM levels were higher, long-term IgM positivity and radiographic damage were more frequent. Overall, after 13 years, pain and fatigue levels remained significant, 5 patients were still treated

Received 14 January 2020; editorial decision 7 May 2020; accepted 12 May 2020; published online May 19, 2020.

The Journal of Infectious Diseases ${ }^{\circledR} \quad$ 2020;222:1740-4

(C) The Author(s) 2020. Published by Oxford University Press for the Infectious Diseases Society of America. All rights reserved. For permissions, e-mail: journals.permissions@oup.com. DOI: 10.1093/infdis/jiaa261 by methotrexate, 3 by TNF-blockers, highlighting long-term Chikungunya-related patient burden.

Conclusions. Suchalong-term persistence ofChikungunyarelated chronic inflammatory rheumatic diseases had not been reported so far. Furthermore, the long-term Chikungunya IgM positivity we observed in some cases might corroborate the hypothesis of residual viral antigen-driven chronic arthritis.

Keywords. anti-CCP negative; Chikungunya; Chikungunya IgM; chronic arthritis; immunosuppressant drugs.

In 2005-2006, Reunion Island suffered a massive Chikungunya outbreak, with an estimated 40\% seroprevalence and 38\% prevalence in a developed but previously nonimmunized population [1], providing a unique opportunity for further clinical and biological understanding of Chikungunya-related joint manifestations [2]. Joint symptoms (arthralgia, arthritis) involve approximately $90 \%$ of the patients in the acute phase of the viral infection. Sixty percent of the patients still suffered from joint symptoms [3] and 39.7\% still showed elevated anti-Chikungunya immunoglobulin (Ig)M levels [4] at 36 and 18 months, respectively, after acute viral infection in 2 Reunion Island cohorts. Several clinical presentations were reported (arthralgia, anticitrullinated peptide autoantibody [ACPA]-negative rheumatoid arthritis-like forms, axial spondyloarthritis (SpA)-like forms, oligoarthritis, tenosynovitis, etc) [5]. In the RHUMATOCHIK study [6], $83 \%$ of 258 patients contacted by phone still reported joint pain 32 months after an initial clinical evaluation by a rheumatologist at the initial stage of Chikungunya infection. However, a clinical re-evaluation by a rheumatologist would be more accurate to assess the nosology, long-term evolution and prognosis of postChikungunya rheumatic diseases.

In this context, we performed a longitudinal rheumatologic re-evaluation (clinical examination, biological parameters, and joint x-rays) after 13 years, in 30 patients who had previously developed post-Chikungunya chronic inflammatory rheumatic diseases, assessed by a rheumatologist, during the 2005-2006 outbreak in Reunion Island.

The main objective was to estimate the proportion of these patients still suffering from Chikungunya-related inflammatory joint symptoms 13 years later. We also aimed at comparing the characteristics of the 2 subgroups of patients defined by the persistence or not of these symptoms after 13 years, to identify prognostic factors.

\section{METHODS}

\section{Recruitment and Study Procedures}

One hundred fifty-nine serologically confirmed Chikungunya patients suffering from chronic musculoskeletal pain were initially referred to a single rheumatologist (A.R.) in Saint-Denis 
teaching hospital (median time interval after the initial viral infection in 2005-2006: 38.5 months). Among them, 73 patients (45.9\%) suffered from classifiable and non-pre-existing chronic inflammatory rheumatic diseases, including $40 \mathrm{pa}-$ tients fulfilling rheumatoid arthritis (RA) American College of Rheumatology (ACR)/European League Against Rheumatism (EULAR) classification criteria, 18 fulfilling the SpA European Spondyloarthropathy Study Group (ESSG) criteria, and 15 fulfilling psoriatic arthritis (PsA) Classification Criteria for Psoriatic Arthritis (CASPAR) criteria [7]. Thirty of these 73 patients were re-evaluated in a longitudinal observational study in 2018-2019 by a second senior rheumatologist (X.G.), after signed informed consent. The study protocol was approved by the research ethics committee of Laveran Military Teaching Hospital, Marseille, France (registration number 2014-PRSimon). Clinical, biological, and joint $\mathrm{x}$-ray (hands, wrists, feet, pelvis) examinations were performed within the range of standard care. Disease activity was assessed using DAS28 score for peripheral arthritides and BASDAI score for axial symptoms of SpA.

Chronic Chikungunya-related inflammatory rheumatic diseases were defined as daily inflammatory joint or spine pain fulfilling (in terms of inflammatory pain, stiffness, joint swelling, and biologic markers) the aforementioned validated classification criteria of RA, SpA, or PsA (sometimes requiring an immunomodulatory treatment and therefore potentially relieved at the time of clinical examination), which initially started concomitantly with the Chikungunya infection and lasted for at least 3 consecutive months [8]. At the second clinical evaluation step in 2018-2019, the patients were then categorized into the symptom persistence group if they still fulfilled the aforementioned definition criteria or into the symptom resolution group if they had no more symptoms nor immunomodulatory drugs linked to the initial Chikungunya-related inflammatory rheumatic disease.

\section{Data Analysis}

Quantitative variables were expressed as means, and qualitative variables were expressed as percentages $\pm 95 \%$ confidence intervals. The $P$ value was $5 \%$. We compared 2 groups of patients (persisting joint symptoms versus no more symptoms), using non-parametric Wilcoxon Mann-Whitney tests for quantitative values and Fisher tests for qualitative values.

\section{RESULTS}

Forty-three of the 73 patients initially classified as Chikungunyarelated classifiable chronic inflammatory rheumatic diseases were lost to follow-up or refused to participate, and 30 were re-evaluated in 2018-2019. Patient characteristics are summarized in Table 1. At the second evaluation time in 2018-2019, the inflammatory joint symptoms persisted in 17 of the 30 patients (mean symptom duration 156 months), whereas 13 patients reported no more pain (mean symptom duration 55.5 months) (Table 1).

\section{DISCUSSION}

In this study, we show for the first time that Chikungunyarelated chronic inflammatory joint symptoms may persist for as long as 13 years after the initial infection. Among 159 patients initially referred for chronic Chikungunya-related chronic musculoskeletal pain, 73 (45.9\%) fulfilled classification criteria of RA or SpA. Thirteen years after the initial viral infection, 43 patients were lost to follow-up, but at least $17 \mathrm{pa}-$ tients were still diagnosed with persistent Chikungunya-related classifiable chronic inflammatory rheumatic diseases (minimal estimations: at least $23.3 \%$ of 73 patients diagnosed with postChikungunya inflammatory rheumatic diseases and $10.7 \%$ of 159 patients referred for post-Chikungunya chronic musculoskeletal pain 38.5 months after the viral infection).

These results provide further insight into the long-term evolution of Chikungunya-related inflammatory joint symptoms. The Chikungunya seroprevalence was estimated to be $40 \%$ in Reunion Island during the 2005-2006 outbreak, and 43\% of the Chikungunya-positive patients still suffered from chronic musculoskeletal pain 24 months after the initial infection (versus $17 \%$ in Chikungunya-negative patients) [1]. Furthermore, if we extrapolate our results, at least $10.7 \%$ of the patients initially referred to a rheumatologist for post-Chikungunya chronic musculoskeletal pain might still suffer from a classifiable chronic inflammatory joint disease after 13 years, and therefore the prevalence of persistent post-Chikungunya inflammatory rheumatic diseases might reach $4.6 \%$ in Chikungunya-seropositive patients $(0.75 \%$ of the general population when taking into account the concomitant expected prevalence of chronic musculoskeletal pain in Chikungunya-negative patients) in Reunion Island, 13 years after the 2005-2006 outbreak. In the literature, the prevalence estimates of persistent Chikungunya-related classifiable chronic inflammatory rheumatic diseases after acute Chikungunya infection ranged from $2 \%$ to $8 \%$ after $15-27.5$ months [9-12]. In other studies not using validated inflammatory rheumatic disease classification criteria, $13.7 \%$ to $64 \%$ persistence rates were reported after 15-36 months [3-6, $8,13]$.

Given the estimated global prevalence of RA $(0.3 \%)$ and $\mathrm{SpA}$ $(0.3 \%)$ in the French population, the expected number of RA and SpA cases in Reunion Island would be approximately 5250 patients. Using the prevalence estimation extrapolated from our results, 5872 additional cases attributable to Chikungunya infection might have occurred since the 2005-2006 outbreak. An epidemiologic study based on anonymized medical records from the national health insurance is on the way and will be an important tool to check this hypothesis, by comparing the prevalence of diagnosed RA and SpA patients in Reunion Island before and after 2005-2006. 


\begin{tabular}{|c|c|c|c|c|}
\hline $\begin{array}{l}\text { Means } \pm 95 \% \mathrm{Cl} \\
\text { or } \\
\mathrm{N}(\text { Percentages } \pm 95 \% \mathrm{Cl})\end{array}$ & $\begin{array}{l}\text { Second Evaluation in 2018- } \\
2019(N=30)\end{array}$ & $\begin{array}{l}\text { Joint Symptom Persist- } \\
\text { ence }(N=17)\end{array}$ & $\begin{array}{l}\text { Joint Symptom } \\
\text { Resolution }(N=13)\end{array}$ & $\begin{array}{l}\text { PValue } \\
\text { (Persistence } \\
\text { Versus Reso- } \\
\text { lution) }\end{array}$ \\
\hline Age (years) & $60.1[57.2-63.1]$ & $58.8[54-63.5]$ & $61.5[56.1-67]$ & .5 \\
\hline Sex, N (\%) & $\begin{array}{l}\text { F: } 20(66[49-83]) \\
M: 10(33[17-51])\end{array}$ & $\begin{array}{l}12(70.6[49-92]) \\
5(29.4[7.7-51])\end{array}$ & $\begin{array}{l}9(69.2[44-94]) \\
4(30.8[5.7-56])\end{array}$ & $\begin{array}{l}1 \\
1\end{array}$ \\
\hline $\begin{array}{l}\text { Initial Rheumatic disease diagnosis in } \\
2005-2006, N(\%)\end{array}$ & $\begin{array}{l}\text { RA: } 19(63[46-80]) \\
\text { SpA: } 5(16.7[3-30]) \\
\text { PsA: } 6(20[6-34])\end{array}$ & $\begin{array}{l}11(64.7[42-87]) \\
2(11.8[0-27]) \\
4(23.5[3-44])\end{array}$ & $\begin{array}{c}8(61.5[35-88]) \\
3(23.1[0.1-46]) \\
2(15.4[0-35])\end{array}$ & $\begin{array}{l}1 \\
.63 \\
.67\end{array}$ \\
\hline Joint symptom duration (months) & $114.4[92-137]$ & 156 [127-185] & $55.5[21-90]$ & $.000005^{* * *}$ \\
\hline Joint symptom evolution, $\mathrm{N}(\%)$ & $\begin{array}{l}\text { Persistence: } 17 \text { (56.7 [39-74]) } \\
\text { Resolution: } 13 \text { (43.3 [26-61]) }\end{array}$ & & & \\
\hline Pain (1-10 VAS) & $4.4[3.3-5.6]$ & $4.8[3.4-6.3]$ & $3.9[2.1-5.7]$ & .49 \\
\hline Fatigue (1-10 VAS) & $4.6[2.7-6.5]$ & $5[2.2-7.8]$ & $3.2[0.6-5.8]$ & .78 \\
\hline Morning stiffness (min) & 17 [9-26] & $18.7[8-29.4]$ & $15.5[2.3-28.7]$ & .38 \\
\hline Number of tender joints & $2.5[0.3-4.8]$ & $4.1[1.1-7]$ & $0.3[0-3.9]$ & .17 \\
\hline Number of swollen joints & $0.4[0-0.8]$ & $0.6[0-1.2]$ & $0.2[0-0.9]$ & .89 \\
\hline DAS28 (ESR) & $3.2[2.7-3.7]$ & $3.6[2.9-4.3]$ & $2.7[1.9-3.5]$ & .058 \\
\hline DAS28 (CRP) & $2.6[2.1-3]$ & $2.9[2.3-3.5]$ & $2.1[1.4-2.8]$ & .13 \\
\hline BASDAI $(S p A+P s A)-N=11$ & 42 [30-53] & 45.4 [29.9-61] & $37.3[20.6-54.1]$ & .83 \\
\hline ESR $(\mathrm{mm})$ & 23 [17-29] & $22.4[14.7-30.1]$ & $23.8[14.5-33.1]$ & .69 \\
\hline $\mathrm{CRP}(\mathrm{mg} / \mathrm{L})$ & $3.9[1.7-6.1]$ & $3.1[0.4-5.9]$ & $5.2[1.6-8.8]$ & .65 \\
\hline $\begin{array}{l}\text { Chikungunya } \lg G(I U) \\
\text { Chikungunya IgM (IU) } \\
\operatorname{lgM}^{+}, N(\%) \\
\operatorname{lgM}^{+} \text {individual values (IU) } \\
\text { lgM }{ }^{+} \text {patient phenotype }\end{array}$ & $\begin{array}{l}1.07[0.74-1.41] \\
0.24[0.04-0.44] \\
4(13.3[0.9-25])\end{array}$ & $\begin{array}{c}1.3[0.8-1.7] \\
0.3[0.1-0.6] \\
3(17.7[0-36]) \\
1.72-1.47-0.45 \\
\text { (RA: } 3 / 3)\end{array}$ & $\begin{array}{c}0.7[0.2-1.3] \\
0.1[0-0.4] \\
1(7.7[0-22]) \\
0.36 \\
(\mathrm{SpA}: 1)\end{array}$ & $\begin{array}{c}.28 \\
.14 \\
1\end{array}$ \\
\hline $\begin{array}{l}\text { ACPA (+), N (\%) } \\
\text { RF (+), N (\%) } \\
\text { ANA (+), N (\%) } \\
\text { Any autoantibody (+), N (\%) }\end{array}$ & $\begin{array}{l}3(10[39-74]) \\
3(10[39-74]) \\
3(10[39-74]) \\
6(20[5.7-34])\end{array}$ & $\begin{array}{c}3(17.7[0-36]) \\
3(17.7[0-36]) \\
3(17.7[0-36]) \\
6(35.3[12-58])\end{array}$ & $\begin{array}{ll}0 & (0) \\
0 & (0) \\
0 & (0) \\
0 & (0)\end{array}$ & $\begin{array}{l}.26 \\
.26 \\
.24 \\
.05^{*}\end{array}$ \\
\hline Uricemia $(\mu \mathrm{mol} / \mathrm{L})$ & 324 [260-369] & 314 [253-375] & $342[273-411]$ & .83 \\
\hline $\begin{array}{l}\text { HLA: } \\
\text { B27 }(+), N(\%) \\
\text { DRB1 }{ }^{*} 01(+), N(\%) \\
\text { DRB1 }{ }^{*} 04(+), N(\%) \\
\text { DRB1 }{ }^{*} 01 \text { or }{ }^{*} 04(+), N(\%)\end{array}$ & $\begin{array}{c}2(6.7[0-15.6]) \\
3(10[0-20.7]) \\
6(20[5-34]) \\
9(30[13.6-46])\end{array}$ & $\begin{array}{c}0(0) \\
1(5.9[0-17]) \\
3(17.7[0-36]) \\
4(23.5[3-44])\end{array}$ & $\begin{array}{l}2(15.4[0-35]) \\
2(15.4[0-35]) \\
3(23.1[0-46]) \\
5(38.5[12-65])\end{array}$ & $\begin{array}{l}.14 \\
.53 \\
.63 \\
.2\end{array}$ \\
\hline $\begin{array}{l}\text { Radiographic structural damage } \\
\text { (joint erosions and/or joint space } \\
\text { narrowing), } \mathrm{N}(\%)\end{array}$ & $7(23.3[8-38])$ & $5(29.4[7.7-51])$ & $2(15.4[0-35])$ & .2 \\
\hline $\begin{array}{l}\text { Radiographic sacroiliitis (NY } \\
\text { criteria), N (\%) }\end{array}$ & $1(3.3[0-0,7])$ & $0(0)$ & 1 (7.7[0-22]) & 1 \\
\hline $\begin{array}{l}\text { Previous treatments (2005-2018) } \\
\text { Methotrexate, N (\%) } \\
\text { Duration (months) } \\
\text { Leflunomide, N (\%) } \\
\text { Sulfasalazine, N (\%) } \\
\text { Hydroxychloroquine, N (\%) } \\
\text { TNF blockers, N (\%) } \\
\text { Duration (months) } \\
\text { Corticosteroids, N (\%) } \\
\text { NSAIDs, N (\%) }\end{array}$ & $\begin{array}{c}23(76.7[62-92])- \\
37[17-61] \\
8(26.7[10.9-42.5]) \\
2(6.7[0-15.6]) \\
1(3.3[0-9.7]) \\
8(26.7[10.9-42.5]) \\
45.8[15-76] \\
11(36.7[19.5-54]) \\
7(23.3[8.2-38.4])\end{array}$ & $\begin{array}{c}14(82[64-100]) \\
45.6[16.9-74.3] \\
5(29.4[7.7-51]) \\
1(5.9[0-17]) \\
0(0) \\
6(35.3[12-58]) \\
33[0-68.4] \\
7(41.2[18-65]) \\
3(17.7[0-36])\end{array}$ & $\begin{array}{c}9(69.2[44-94]) \\
22[0-68.3] \\
3(23.1[0-46]) \\
1(7.7[0-22]) \\
1(7.7[0-22]) \\
2(15.4[0-35]) \\
84[22.6-145.4] \\
4(30.8[5.7-56]) \\
4(30.8[5.7-56])\end{array}$ & $\begin{array}{l}.54 \\
.32 \\
1 \\
1 \\
.4 \\
.67 \\
.23 \\
.3 \\
.57\end{array}$ \\
\hline $\begin{array}{l}\text { Current treatments (2018-19) } \\
\text { Methotrexate, N (\%) } \\
\text { TNF blockers, N (\%) }\end{array}$ & $\begin{array}{c}5(16.7[3.4-30]) \\
3(10[0-20.7])\end{array}$ & $\begin{array}{l}5(29.4[7.7-51]) \\
3(17.7[0-36])\end{array}$ & $\begin{array}{l}0(0) \\
0(0)\end{array}$ & $\begin{array}{l}.12 \\
.26\end{array}$ \\
\hline
\end{tabular}

Abbreviations: ACPA, anticitrullinated peptide autoantibodies; ANA, antinuclear antibody; BASDAl, bath ankylosing spondylitis disease activity index; Cl, confidence interval; CRP, C-reactive protein; DAS28, 28 joint disease activity score; ESR, erythrocyte sedimentation rate; F, female; HLA, human leukocyte antigen; Ig, immunoglobulin; M, male; NSAIDs, nonsteroidal anti-inflammatory drugs; NY, New York; PsA, psoriatic arthritis (Classification Criteria for Psoriatic Arthritis [CASPAR] criteria); RA, rheumatoid arthritis (American College of Rheumatology/European League Against Rheumatism [ACR/EULAR] criteria); RF, rheumatoid factor (the same 3 patients were both ACPA and RF positive); SpA, spondyloarthritis (European Spondyloarthropathy Study Group [ESSG] criteria); TNF, tumor necrosis factor; VAS, visual analog scale.

${ }^{a}$ Results are expressed by means or percentages with $95 \%$ Cls. For comparisons between persistence and resolution groups, means were compared using Wilcoxon-Mann Whitney tests, whereas percentages were compared using Fisher tests; $P=.05 .{ }^{*} P<.05 ;{ }^{*} *{ }^{*} P<.001$. 
The mean Chikungunya IgM and IgG levels tended to be higher in the patients with persistent joint symptoms, and 3 patients remained Chikungunya- IgM positive 13 years after the infection in the Persistence group (versus 1 in the Resolution group). The Chikungunya IgG levels had already been reported to be associated with more frequent joint symptom persistence after 24 months [1], and a long-term persistence of Chikungunya IgM positivity had also been reported after 18 months [4]. This might be linked with viral antigen persistence in "sanctuary" tissues such as synovial macrophages of infected patients, potentially causing chronic joint inflammation [2]. We also observed more radiographic joint damage in the group with persistence of the symptoms (29\% versus $15 \%$, $P=.2$ ). It is notable that, among the 5 patients with persistent joint symptoms and radiographic damage, 2 still showed significantly positive Chikungunya IgM levels 13 years after the initial infection. Overall, we observed radiographic damage evocative of inflammatory joint diseases in 7 of 30 of our patients ( 5 of 19 in RA patients) after 13 years, the proportion of which was potentially reduced/limited by the use of immunomodulatory drugs. The previously reported prevalence estimates of radiographic damage in Chikungunya-related inflammatory joint diseases ranged from $0 \%$ after 6 [14] and 15 months [10] to $57 \%$ and $81 \%$ after 10 and 24 months [15].

The overall proportion of positive autoantibodies was relatively low (6 of 30 patients, including 3 of 19 RA patients fulfilling the ACR/EULAR criteria)-lower than the expected prevalence of autoantibodies in established inflammatory rheumatic diseases (for instance, 50\%-75\% of established RA patients are ACPA positive)—consistent with previous studies on the topic $[6,8-10]$, suggesting widely autoantibody and peptide citrullination-independent pathophysiological mechanisms [8] such as viral antigen persistence in tissue sanctuaries [2], virus-induced epigenetic modifications of synovial macrophages (inducing more invasive and proinflammatory phenotypes), or molecular mimicry through the persistence of Chikungunya-specific antibodies, which might cross-react with host synovial autoantigens. More importantly, the autoantibodies were positive from the beginning, and no seroconversion was observed during the 13-year follow-up, as was reported in previous studies $[8,9]$. We observed antinuclear antibody or ACPA/rheumatoid factor autoantibody positivity in 6 of 17 patients with persistent symptoms after 13 years versus 0 of 13 patients in the Resolution group ( $P=.05$, mean symptom durations: 156 versus 55.5 months). Although the Chikungunya infection does not seem to induce de novo autoantibody production, our results suggest that baseline autoantibody-positive patients - who are generally more at risk of developing chronic inflammatory joint diseases-might be prone to develop more prolonged (beyond 13 years) Chikungunya-related chronic inflammatory rheumatic diseases as well. This association had not been reported in previous shorter term follow-up studies. The relatively moderate difference in terms of mean pain levels we observed between the 2 groups (Table 1 ) could be explained by the fact that the patients with persistent inflammatory rheumatic diseases were examined during the chronic phase (not during disease flares) and that 8 of 17 patients in this group were still treated by immunomodulatory drugs. More importantly, even though the Resolution group patients reported some level of residual pain (mostly due to degenerative conditions such as osteoarthritis), they did not fulfill the classification criteria of inflammatory rheumatic diseases nor required immunomodulatory treatments anymore. It is logical that the difference between the 2 groups regarding the DAS28 inflammatory activity score was more pronounced $(P=.058)$.

After 13 years, 8 of 30 patients were still treated by methotrexate, 3 of 30 by TNF blockers (all belonging to the joint symptom persistence group), and the mean pain and fatigue scores remained relatively high, highlighting a significant burden in terms of general health.

Our study had some limitations. It is notable that the sample size was relatively small, due to lost to follow-up patients, introducing an unavoidable attrition bias and a low absolute number of cases, which underpowered the statistical analyses we performed. However, our study showed interesting trends concerning an unexpectedly long-term Chikungunya IgM positivity and a statistically significant association between autoantibody baseline positivity and a longer term symptom persistence. These results will require further confirmation in larger scale studies. Furthermore, the minimal estimated prevalence of joint symptom persistence after 13 years (17 of 73 patients with chronic inflammatory joint diseases, 17 of 159 patients with post-Chikungunya chronic musculoskeletal pain) might have been underestimated due to the lost to follow-up patients, even though these patients could have been less likely to suffer from persistent joint symptoms. The study was monocentric, and all the patients were initially referred to a single rheumatologist in a university hospital, which might have introduced a selection bias, and, conversely, a potential overrepresentation of severe and refractory inflammatory rheumatic diseases. We also focused on a specific population, defined by validated classification criteria of inflammatory rheumatic diseases, and therefore did not consider the full spectrum of Chikungunya-related long-term sequelae. Furthermore, no specific epidemiologic data regarding RA nor SpA prevalence exist for the Reunion Island population, making it difficult to evaluate the real impact of Chikungunya infection. An ongoing epidemiologic study using the French national health insurance system records will help determine whether (and to what extent) this prevalence actually increased in Reunion Island after the 2005-2006 Chikungunya outbreak.

The important strength of our study is the follow-up duration (13 years after viral infection), which is unprecedented in the literature (the previous longest term published follow-up data 
did not exceed 40 months). Unlike all other studies on the topic, the second evaluation consisted of a standardized clinical, biological, and radiographic evaluation by a senior rheumatologist, therefore reducing the risk of biases inherent to telephonic interviews [6] or retrospective data analyses (potential overestimation of clinical parameters such as joint swelling, potential misclassification between degenerative and inflammatory rheumatic diseases).

\section{CONCLUSIONS}

The present results concerning the long-term evolution of Chikungunya serology, autoantibody status, and radiographic parameters provide further insight into the natural history Chikungunya-related chronic inflammatory rheumatic diseases and lend credibility to mechanistic hypotheses such as a potential persistent viral antigen-mediated immune stimulation.

\section{Notes}

Acknowledgments. The present study was a follow-up of the study published by Pr F. Simon and Dr E. Javelle (Laveran Military Hospital, Marseille, France) in 2014 [7]. Chikungunya serological tests were performed in Reunion University Hospital by Pr MC Jaffar-Bandjee (CNR associé arbovirus).

Author contributions. X. G. and P. G. contributed to conceptualization, methodology, manuscript redaction, and manuscript review. X. G. and A. R. contributed to patient clinical evaluation and data collection. X. G. contributed to statistical analyses.

Potential conflicts of interest. All authors: No reported conflicts of interest. All authors have submitted the ICMJE Form for Disclosure of Potential Conflicts of Interest.

\section{References}

1. Gérardin P, Fianu A, Michault A, et al. Predictors of Chikungunya rheumatism: a prognostic survey ancillary to the TELECHIK cohort study. Arthritis Res Ther 2013; 15:R9.

2. Hoarau JJ, Jaffar Bandjee MC, Krejbich Trotot P, et al. Persistent chronic inflammation and infection by Chikungunya arthritogenic alphavirus in spite of a robust host immune response. J Immunol 2010; 184:5914-27.

3. Schilte C, Staikowsky F, Staikovsky F, et al. Chikungunya virus-associated long-term arthralgia: a 36-month prospective longitudinal study. PLoS Negl Trop Dis 2013; 7:e2137.

4. Borgherini G, Poubeau P, Jossaume A, et al. Persistent arthralgia associated with chikungunya virus: a study of 88 adult patients on Reunion Island. Clin Infect Dis 2008; 47:469-75.
5. Rodríguez-Morales AJ, Cardona-Ospina JA, Fernanda Urbano-Garzón S, Sebastian Hurtado-Zapata J. Prevalence of post-Chikungunya infection chronic inflammatory arthritis: a systematic review and meta-analysis. Arthritis Care Res (Hoboken) 2016; 68:1849-58.

6. Bouquillard E, Fianu A, Bangil M, et al. Rheumatic manifestations associated with Chikungunya virus infection: a study of 307 patients with 32-month follow-up (RHUMATOCHIK study). Joint Bone Spine 2018; 85:207-10.

7. Javelle E, Ribera A, Degasne I, Gaüzère B-A, Marimoutou C, Simon F. Specific management of post-Chikungunya rheumatic disorders: a retrospective study of 159 cases in Reunion Island from 2006-2012. PLoS Negl Trop Dis 2015; 9:e0003603.

8. Chang AY, Martins KAO, Encinales L, et al. Chikungunya arthritis mechanisms in the Americas: a cross-sectional analysis of Chikungunya arthritis patients twenty-two months after infection demonstrating no detectable viral persistence in synovial fluid. Arthritis Rheumatol 2018; 70:585-93.

9. Chopra A, Anuradha V, Ghorpade R, Saluja M. Acute Chikungunya and persistent musculoskeletal pain following the 2006 Indian epidemic: a 2-year prospective rural community study. Epidemiol Infect 2012; 140:842-50.

10. Mathew AJ, Goyal V, George E, Thekkemuriyil DV, Jayakumar B, Chopra A; Trivandrum COPCORD Study Group. Rheumatic-musculoskeletal pain and disorders in a naïve group of individuals 15 months following a Chikungunya viral epidemic in south India: a population based observational study. Int J Clin Pract 201 1; 65:1306-12.

11. van Aalst M, Nelen CM, Goorhuis A, Stijnis C, Grobusch MP. Long-term sequelae of chikungunya virus disease: A systematic review. Travel Med Infect Dis 2017; 15:8-22.

12. Essackjee K, Goorah S, Ramchurn SK, Cheeneebash J, Walker-Bone K. Prevalence of and risk factors for chronic arthralgia and rheumatoid-like polyarthritis more than 2 years after infection with Chikungunya virus. Postgrad Med J 2013; 89:440-7.

13. Sissoko D, Malvy D, Ezzedine K, et al. Post-epidemic Chikungunya disease on Reunion Island: course of rheumatic manifestations and associated factors over a 15-month period. PLoS Negl Trop Dis 2009; 3:e389.

14. Chopra A, Anuradha V, Lagoo-Joshi V, Kunjir V, Salvi S, Saluja M. Chikungunya virus aches and pains: an emerging challenge. Arthritis Rheum 2008; 58:2921-2.

15. Bouquillard E, Combe B. A report of 21 cases of rheumatoid arthritis following Chikungunya fever. A mean follow-up of two years. Joint Bone Spine 2009; 76:654-7. 